Case Report

\title{
Case Report of Lambert Eaton Myasthenic Syndrome in a Patient with Small Cell Lung Cancer on Immune Checkpoint Inhibitor Therapy
}

Collin J. Anderson ${ }^{1,}{ }^{*}$, Amanda C. Guidon ${ }^{2}$, Farrah B. Khan ${ }^{3}$, Alissa A. Thomas ${ }^{4}$, Carolyne Riehle ${ }^{1}$, Michael K. Hehir ${ }^{1}$, Noah A. Kolb ${ }^{1, *}$

1. Department of Neurology, University of Vermont Larner College of Medicine, Burlington, VT, USA; E-Mails: collin.anderson@med.uvm.edu; carolyne.riehle@uvmhealth.org; michael.hehir@uvmhealth.org; noah.kolb@uvmhealth.org

2. Department of Neurology, Massachusetts General Hospital, Harvard Medical School, Boston, MA, USA; E-Mail: aguidon@partners.org

3. Department of Medicine, Division of Medical Oncology, University of Vermont Larner College of Medicine, Burlington, VT, USA; E-Mail: farrah.khan@uvmhealth.org

4. Department of Neurology, Division of Neuro-Oncology, University of Vermont Larner College of Medicine, Burlington, VT, USA; E-Mail: alissa.thomas@uvmhealth.org

* Correspondence: Collin J. Anderson and Noah A. Kolb; E-Mails: collin.anderson@med.uvm.edu; noah.kolb@uvmhealth.org

Academic Editor: Bart Ellenbroek

OBM Neurobiology

2021, volume 5, issue 1

doi:10.21926/obm.neurobiol.2101086
Received: October 28, 2020

Accepted: January 20, 2021

Published: January 31, 2021

\begin{abstract}
Lambert Eaton myasthenic syndrome (LEMS) is a rare autoimmune neuromuscular junction disorder involving loss of functional pre-synaptic P/Q-type voltage-gated calcium channels. Many cases occur as a paraneoplastic disorder, often in small cell lung cancer (SCLC). Recently, immune checkpoint inhibitors (ICI) have emerged as treatment of choice for various malignancies. While generally well tolerated, certain ICI-treated patients experience neurologic immune-related adverse events (irAEs). Here, we explore therapeutic and diagnostic conundrums from the unclear etiology (paraneoplastic vs. irAE) of a case of LEMS in a patient with SCLC treated with ICl therapy. A 62-year-old female patient with SCLC was referred to EMG laboratory with 7 weeks of progressive weakness, shortness of breath and
\end{abstract}

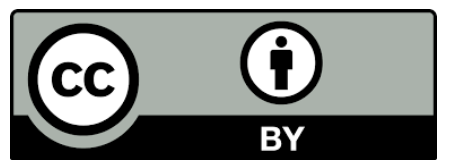

(C) 2021 by the author. This is an open access article distributed under the conditions of the Creative Commons by Attribution License, which permits unrestricted use, distribution, and reproduction in any medium or format, provided the original work is correctly cited. 
dysphagia. Due to tumor progression, immunotherapy with pembrolizumab was initiated five months prior to presentation. On examination, she had mild non-fatigable right-sided ptosis and diplopia, normal bulbar strength, and proximal greater than distal weakness of lower greater than upper extremities. Her reflexes were 2-/4 throughout, with left biceps reflex facilitating after 30 seconds of exercise. On nerve conduction studies (NCS), there was an amplitude increase in multiple nerves including the left median nerve (160\%) and left ulnar nerve $(370 \%)$ after 10 seconds of exercise. Paraneoplastic panel came back with elevated LEMS-related anti-P/Q-type voltage gated calcium channel antibodies at $0.19 \mathrm{nmol} / \mathrm{L}$ (normal: $\leq 0.02 \mathrm{nmol} / \mathrm{L}$ ). This case illustrates the diagnostic and therapeutic challenges that surround LEMS in SCLC patients on immunotherapy. Diagnosis hinges on clinical presentation, motor NCS, and antibody testing while determination of the etiology (paraneoplastic vs ICl related LEMS) is more complex and may affect selection of the correct treatment. Therapy for IClrelated neuromuscular irAEs depends on symptom severity, but typically should include holding immunotherapy and administration of high dose corticosteroids as first line treatment with possible addition of IVIg and plasmapheresis. This differs from the common first line treatment for paraneoplastic LEMS, highlighting the importance of understanding of the etiology. Further research is needed to better understand optimal management.

\section{Keywords}

Lambert-Eaton myasthenic syndrome; immune-related adverse events; immune checkpoint inhibitors; EMG; needle electromyography

\section{Introduction}

Immunotherapy, including immune checkpoint inhibitors (ICls), has emerged as a novel treatment for a variety of malignancies. These drugs block checkpoint proteins from binding with their ligand/receptor which disinhibits $\mathrm{T}$ lymphocytes and allows them to kill cancer cells. Pembrolizumab is a humanized monoclonal antibody against one of these checkpoint proteins, programmed death 1 (PD-1). Pembrolizumab is indicated for treatment of multiple malignancies, including PD-L1-expressing small cell lung cancer (SCLC) [1]. While generally well-tolerated, up to $12 \%$ of ICl-treated patients experience neurologic immune-related adverse events (irAEs), including encephalitis/meningitis, neuropathies, myositis, and myasthenia gravis (MG) $[2,3]$. Lambert Eaton myasthenic syndrome (LEMS) has rarely been reported [4, 5]. Importantly, the treatment approach to neuromuscular irAEs can differ from that of the classical forms of the same neuromuscular disease. Here, we explore the diagnostic and therapeutic challenges of LEMS in a patient with SCLC treated with $\mathrm{ICl}$ therapy.

\section{Case Presentation}

A 62-year-old female patient presented with recurrent stage IVA SCLC and was referred for EMG with 7 weeks of progressive weakness. Notably, this weakness was not present upon initial presentation of SCLC. 
This patient was chosen for our study based on her symptoms, which began after 8 cycles ( 5 months) of pembrolizumab treatment, with the drug being held at onset of weakness. She described difficulty rising from a chair, followed by instability requiring a walker and wheel chair and difficulty completing activities of daily living. Four weeks after onset, she developed shortness of breath at rest and mild dysphagia. She denied any visual symptoms, fatigability, or diurnal variation in her weakness.

On examination, our patient had mild non-fatigable right-sided ptosis without eye closure weakness. Eye movements appeared normal; however, she complained of diplopia in neutral gaze that did not worsen with sustained up or lateral gaze. She could whistle, puff her cheeks against resistance and was wheelchair-bound. Medical Research Council (MRC) strength: Neck flexion 4+/5, shoulder abduction and hip flexion 4-/5, distal strength $4+/ 5$. Her reflexes were $1+/ 4$ throughout, however the left biceps reflex facilitated to $2+/ 4$ with 30 seconds of exercise. The remainder of her neurologic examination was normal.

Motor nerve conduction studies showed low amplitude compound muscle action potential (CMAP) with normal latencies and conduction velocities in the right peroneal, tibial, median, and left median and ulnar nerves. Slow $3-\mathrm{Hz}$ repetitive nerve stimulation revealed decrement of $44 \%$ in the right ulnar-abductor digiti minimi (ADM) and $37 \%$ in the right median-abductor pollicis brevis (APB) nerve-muscle combinations (Figure 1). With 10 seconds of exercise there was $370 \%$ and $160 \%$ post exercise facilitation of the baseline left ulnar and median CMAP amplitudes, respectively (Figure 1). There was mild evidence of carpal tunnel syndrome, which had been previously diagnosed. All sensory nerve conduction studies were normal. Concentric needle EMGs of the right first dorsal interossei, biceps, deltoid, triceps, and pronator teres were normal.

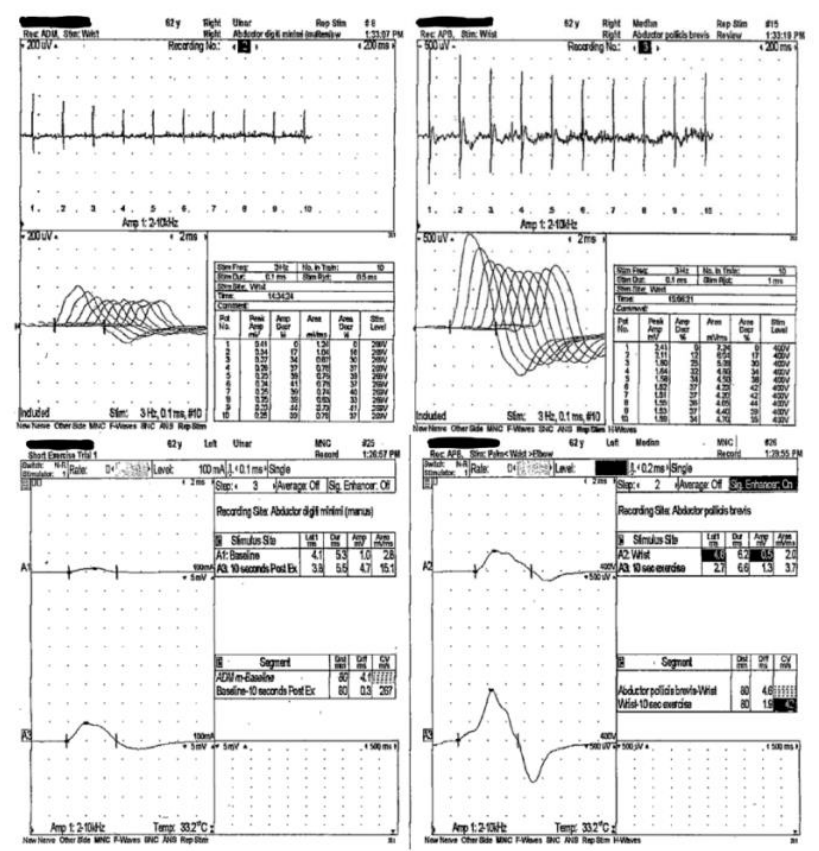

Figure $13 \mathrm{~Hz}$ repetitive nerve stimulation demonstrated CMAP amplitude decrement of $44 \%(0.4 \mathrm{mV}$ to $0.2 \mathrm{mV}$ ) in right ulnar nerve (top left of figure) and $37 \%$ ( $2.4 \mathrm{mV}$ to $1.5 \mathrm{mV}$ ) in right median nerve (top right of figure). EMG demonstrated post-exercise CMAP amplitude increases of $370 \%$ ( $1.0 \mathrm{mV}$ to $4.7 \mathrm{mV}$ ) in left ulnar nerve (bottom left of figure) and $160 \%(0.5 \mathrm{mV}$ to $1.3 \mathrm{mV})$ in left median nerve (bottom right of figure). 
Given the history and electrodiagnostic findings, LEMS was suspected. Laboratory evaluation identified elevated anti-P/Q-type voltage gated calcium channel (VGCC) antibodies at $0.19 \mathrm{nmol} / \mathrm{L}$ (normal: $\leq 0.02 \mathrm{nmol} / \mathrm{L}$ ). Anti-acetylcholine receptor $(\mathrm{AChR})$ binding, blocking and modulating, muscle-specific kinase (MuSK), TSH and N-type VGCC antibodies, CK, and myomarker panel were all normal or negative. The presence of concurrent irAE-related, antibody-negative MG was considered given the decremental response on her EMG (which can be seen in LEMS), mild ptosis, diplopia (although neither was fatigable) and shortness of breath which are atypical symptoms for LEMS. While the lack of clear fatigability commonly seen in MG was not observed, concurrent irAE related MG could not be fully excluded.

Pembrolizumab had already been held at the time of neurologic evaluation. She was started on $1 \mathrm{mg} / \mathrm{kg}$ daily of prednisone while awaiting antibody results and 3,4 DAP insurance approval. Two weeks of corticosteroids yielded no benefit and the patient independently discontinued them without a taper. Shortly thereafter, 3,4 DAP was initiated at 10mg TID with minimal improvement after two weeks. She then started triweekly IVIg with a dramatic and sustained improvement in her symptoms. The patient then decided to stop 3,4 DAP. Within hours both she and her daughter noted reduced strength and decreased mobility. She restarted 3,4 DAP the following day and within hours returned to her new baseline. At her most recent follow up performed over the telephone, she denied any upper or lower extremity weakness, dysarthria, dysphagia, diplopia, ptosis, or shortness of breath and reported minimal leg weakness for the three days preceding her IVIg treatments. Her cancer therapy was changed to paclitaxel with good response, and pembrolizumab was not restarted.

\section{Discussion}

We present a case of a 62-year-old woman with advanced stage SCLC who presented with LEMS while undergoing pembrolizumab treatment. In this setting, determining the LEMS etiology (i.e. paraneoplastic vs. irAE) is critical to enact the right treatment plan.

LEMS is a rare autoimmune neuromuscular junction disorder involving the loss of functional presynaptic P/Q-type VGCCs [6]. Over $60 \%$ of cases occur as a paraneoplastic disorder that is most frequently associated with SCLC $[6,7]$. On motor nerve stimulation, a $60 \%$ increment in compound muscle action potential (CMAP) amplitude following 10 seconds of sustained exercise of a muscle with a low baseline CMAP amplitude is considered $97 \%$ sensitive for LEMS and $99 \%$ specific for LEMS [8]. Autoantibodies to P/Q-type VGCCs show $91 \%$ sensitivity and nearly $100 \%$ specificity for LEMS diagnosis [9].

The mainstay of therapy for classic LEMS is treatment of the underlying malignancy and 3,4 DAP, which augments acetylcholine release at the neuromuscular junction [10]. Refractory patients often require immunosuppressants [11]. IVlg at $2 \mathrm{~g} / \mathrm{kg}$ over 3-5 days followed by $1 \mathrm{~g} / \mathrm{kg}$ every 3-4 weeks, is typical first line treatment for refractory weakness $[12,13]$. While steroids can improve symptoms [14], the response is typically less robust than seen in other neuromuscular disorders [15]. Plasma exchange is reserved for non-responders or those with contraindications to IVIg [16].

There are limited reports of $\mathrm{ICI}$-related LEMS and no consensus on optimal treatment $[4,17,18]$. In general, management of neuromuscular irAEs depends on symptom severity [19]. For very mild symptoms, immunotherapy may continue with close serial monitoring. If symptoms are moderate, severe, or life-threatening, immunotherapy is immediately held. The next steps in the work up 
depends on the specific irAE (muscle biopsy, EMG etc.), and consultation with a neuromuscular or irAE expert neurologist is suggested [20]. Counter to classic LEMS, the first line treatment for most neuromuscular irAEs is high dose corticosteroids [21]. For some ICl-related neuromuscular toxicities, IVIg and plasmapheresis are indicated as well [22]. Due to the paucity of cases of ICl-related LEMS, it remains unclear if ICl-related LEMS will be highly responsive to 3,4 DAP without the need for other adjunctive immunosuppressive therapy (as is typical for paraneoplastic LEMS), or if they will behave more like other ICl-related neuromuscular toxicities and require steroids and/or addition immunosuppressive therapies. Notably, in the two previous case reports of irAE LEMS, the response to steroids was limited, counter to most irAEs and one patient who failed IVIG therapy required rituximab $[17,18]$. Although the numbers are very small it does raise some questions about the pathophysiology of irAEs LEMS and consequently the optimal management of resilient immunemediated LEMS.

In cases of LEMS in ICI SCLC patients, the etiology matters because LEMS treatment may have effects on the efficacy and selection of ongoing cancer treatment for two reasons. Firstly, in immunogenic malignancies like melanoma and lung cancer, there is limited and sometimes contradictory evidence that muting the immune response against cancer with steroids may affect cancer outcomes [22]. In NSCLC, there is evidence that steroids prior to and during immunotherapy worsens outcomes [23]. In other malignancies, studies show no survival or outcome difference with steroid use to manage irAEs [22]. Secondly, patients with paraneoplastic LEMS can continue ICI therapy while those with irAE-related LEMS should stop immunotherapy and may later consider rechallenge. Results for re-challenges with $\mathrm{ICl}$ therapies after neuromuscular irAE are mixed. Some experts recommend retrial with a different class of $\mathrm{ICI}[2,3,20,24-26]$.

We predict that as other cases are described, it may remain difficult to determine the etiology of LEMS in SCLC patients treated with ICI therapy. Careful evaluation of antibody status and timing of symptoms may help. 91\% of those with paraneoplastic LEMS have P/Q-type VGCC antibodies [27, 28]. The absence of antibodies should raise suspicion for ICl-related etiology, as other neuromuscular irAEs show lower incidence of antibodies compared to classic forms of the disease (i.e. $90 \%$ AchR antibodies in generalized classic MG [29], vs 66\% for ICl-related MG) [30]. The time course may also provide clues. In classic LEMS, the symptoms often precede the cancer diagnosis [27] while the median time from immunotherapy to neuromuscular irAEs is 19 weeks (range 1-115) [31]. Evaluation of electrophysiologic findings and work up for concurrent neuromuscular irAEs is critical. Treatment should be guided by these findings (Figure 2). 


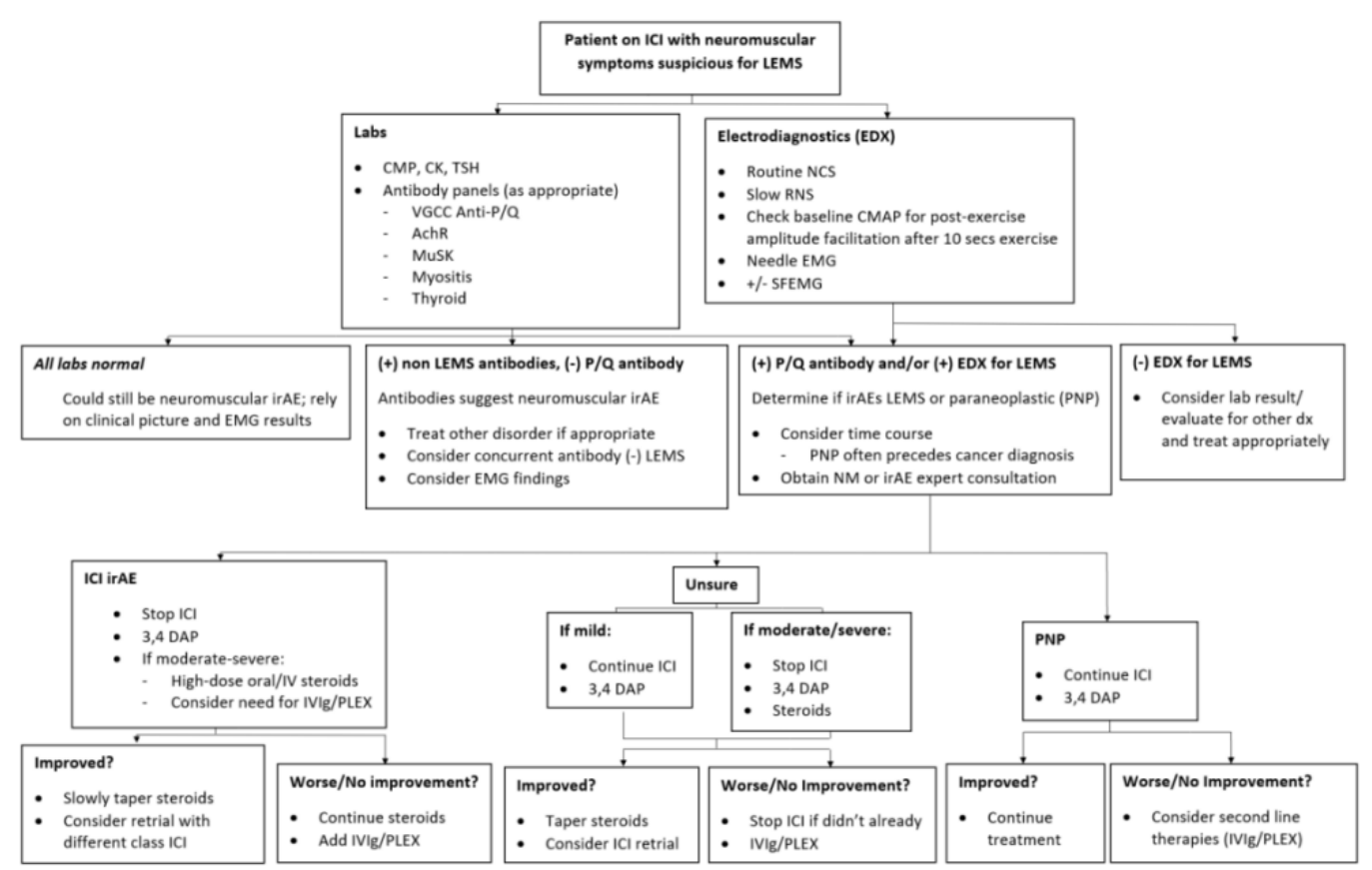

Figure 2 Flowchart demonstrating diagnostic and treatment algorithm for patients on $\mathrm{ICI}$ therapy presenting with neuromuscular symptoms suspicious for LEMS.

The etiology of our patients' LEMS remains unclear. The presence of P/Q-type VGCC antibodies and lack of steroid response might argue for a paraneoplastic cause while the delayed onset of symptoms favors ICl-related LEMS. Further complicating the case, although her AchR antibodies were negative, she had shortness of breath and diplopia (atypical for LEMS), raising the possibility of concurrent ICl-related antibody negative MG which would also be treated with IVIg and corticosteroids. Patients with multiple irAEs are commonly reported [20].

This case illustrates the diagnostic and therapeutic challenges that surround LEMS in SCLC patients on immunotherapy. Further research is needed to develop consensus and evidence-based guidelines for treatment.

\section{Abbreviations}

AchR: Acetylcholine Receptor

CMAP: Compound Muscle Action Potential

CMP: Complete Metabolic Panel

EMG: Electromyography

ICI: Immune Checkpoint Inhibitor

irAE: immune-related Adverse Event

IVIg: Intravenous Immune Globulin

MuSK: Muscle-Specific Kinase

NCS: Nerve Conduction Study

PLEX: Plasma Exchange

RNS: Repetitive Nerve Stimulation

SFEMG: Single-fiber Electromyography

TSH: Thyroid Stimulating Hormone 
VGCC: Voltage Gated Calcium Channel

\section{Author Contributions}

Farrah B. Khan, Alissa A. Thomas, Carolyne Riehle and Michael K. Hehir: analyze the data and edit the manuscript. Noah A. Kolb: design the study, analyze the data, prepare, and edit the manuscript. $\mathrm{He}$ is the second corresponding and archival author.

\section{Competing Interests}

The authors have declared that no competing interests exist.

\section{References}

1. Ott PA, Elez E, Hiret S, Kim DW, Morosky A, Saraf S, et al. Pembrolizumab in patients with extensive-stage small-cell lung cancer: Results from the phase Ib KEYNOTE-028 study. J Clin Oncol. 2017; 35: 3823-3829.

2. Dubey D, David WS, Reynolds KL, Chute DF, Clement NF, Cohen JV, et al. Severe neurological toxicity of immune checkpoint inhibitors: Growing spectrum. Ann Neurol. 2020; 87: 659-669.

3. Reynolds KL, Guidon AC. Diagnosis and management of immune checkpoint inhibitorassociated neurologic toxicity: Illustrative case and review of the literature. Oncologist. 2019; 24: 435-443.

4. Nakatani Y, Tanaka N, Enami T, Minami S, Okazaki T, Komuta K. Lambert-eaton myasthenic syndrome caused by nivolumab in a patient with squamous cell lung cancer. Case Rep Neurol. 2018; 10: 346-352.

5. Jiwa N, Lim-Fat MJ, Chukwueke U, Sands J, Doughty C. Lambert-eaton myasthenic syndrome in the setting of immune checkpoint inhibitor treatment of small cell lung cancer. Rochester, $\mathrm{MN}$ : AANEM Myasthenia Gravis Foundation of America; 2019.

6. Schoser B, Eymard B, Datt J, Mantegazza R. Lambert-eaton myasthenic syndrome (LEMS): A rare autoimmune presynaptic disorder often associated with cancer. J Neurol. 2017; 264: 18541863

7. Rooke ED, Eaton LM, Lambert EH, Hodgson $\mathrm{CH}$. Myasthenia and malignant intrathoracic tumor. Med Clin North Am. 1960; 44: 977-988.

8. Oh SJ, Kurokawa K, Claussen GC, Ryan Jr HF. Electrophysiological diagnostic criteria of LambertEaton myasthenic syndrome. Muscle Nerve.2005; 32: 515-520.

9. Motomura M, Lang B, Johnston I, Palace J, Vincent A, Newsom-Davis J. Incidence of serum anti$\mathrm{P} / \mathrm{O}$-type and anti-N-type calcium channel autoantibodies in the Lambert-Eaton myasthenic syndrome. J Neurol Sci. 1997; 147: 35-42.

10. Oh SJ, Claussen GG, Hatanaka Y, Morgan MB. 3,4-Diaminopyridine is more effective than placebo in a randomized, double-blind, cross-over drug study in LEMS. Muscle Nerve. 2009; 50: 795-800.

11. Benatar M. Myasthenia gravis and myasthenic disorders. Neurology. 2012; 81: 99.

12. Bain PG, Motomura M, Newsom-Davis J, Misbah SA, Chapel HM, Lee ML, et al. Effects of intravenous immunoglobulin on muscle weakness and calcium-channel autoantibodies in the Lambert-Eaton myasthenic syndrome. Neurology. 1996; 47: 678-683. 
13. Keogh M, Sedehizadeh S, Maddison P. Treatment for Lambert-Eaton myasthenic syndrome. Cochrane Database Syst Rev. 2011; 2011: CD003279.

14. Streib EW. Eaton-Lambert myasthenic syndrome: Long-term treatment of three patients with prednisone. Ann Neurol. 1981; 10: 448-453

15. Maddison P, Lang B, Mills K, Newsom-Davis J. Long term outcome in Lambert-Eaton myasthenic syndrome without lung cancer. J Neurol Neurosurgery Psychiatry. 2001; 70: 212-217.

16. Dau PC, Denys EH. Plasmapheresis and immunosuppressive drug therapy in the Eaton-Lambert syndrome. Ann Neurol. 1982: 11: 570-575.

17. Agrawal K, Agrawal N. Lambert-Eaton myasthenic syndrome secondary to nivolumab and ipilimumab in a patient with small-cell lung cancer. Case Rep Neurol Med. 2019; 2019: 53532020.

18. Gill AJ, Gandhy S, Lancaster E. Nivolumab-associated Lambert-Eaton myasthenic syndrome and cerebellar dysfunction in a patient with a neuroendocrine tumor. Muscle Nerve. 2020.

19. Thompson JA, Schneider BJ, Brahmer J, Andrews S, Armand P, Bhatia S, et al. Management of immunotherapy-related toxicities, version 1.2019, NCCN clinical practice guidelines in oncology. J Natl Compr Canc Netw. 2019; 17: 255-289.

20. Guidon AC. Lambert-Eaton myasthenic syndrome, botulism, and immune checkpoint inhibitorrelated myasthenia gravis. Continuum. 2019; 25: 1785-1806.

21. Spain L, Diem S, Larkin J. Management of toxicities of immune checkpoint inhibitors. Cancer Treat Rev. 2016; 44: 51-60.

22. Horvat TZ, Adel NG, Dang TO, Momtaz P, Postow MA, Callahan MK, et al. Immune-related adverse events, need for systemic immunosuppression, and effects on survival and time to treatment failure in patients with melanoma treated with ipilimumab at memorial sloan kettering cancer center. J Clin Oncol. 2015; 33: 3193-3198.

23. Arbour KC, Mezquita L, Long N, Rizvi H, Auclin E, Ni A, et al. Impact of baseline steroids on efficacy of programmed cell death-1 and programmed death-ligand 1 blockade in patients with non-small-cell lung cancer. J Clin Oncol. 2018; 36: 2872-2878.

24. Teply BA, Lipson EJ. Identification and management of toxicities from immune checkpointblocking drugs. Oncology. 2014; 28: 30-38.

25. Tarhini A. Immune-mediated adverse events associated with ipilimumab CTLA-4 blockade therapy: The underlying mechanisms and clinical management. Scientifica. 2013; 2013: 857519.

26. Zalewski NL, Lennon VA, Lachance DH, Klein CJ, Pittock SJ, Mckeon A. P/Q- and N-type calciumchannel antibodies: Oncological, neurological, and serological accompaniments. Muscle Nerve. 2016; 54: 220-227.

27. Titulaer MJ, Wirtz PW, Willems LN, Van Kralingen KW, Smitt PA, Verschuuren JJ. Screening for small-cell lung cancer: A follow-up study of patients with Lambert-Eaton myasthenic syndrome. J Clin Oncol. 2008; 26: 4276-4281.

28. Hülsbrink R, Hashemolhosseini S. Lambert-Eaton myasthenic syndrome-diagnosis, pathogenesis and therapy. Clin Neurophysiol. 2014; 125: 2328-2336.

29. Koon HC, Lachance DH, Harper CM, Lennon VA. Frequency of seronegativity in adult-acquired generalized myasthenia gravis. Muscle Nerve. 2007; 36: 651-658.

30. Safa $H$, Johnson DH, Trinh VA, Rodgers TE, Lin H, Suarez-Almazor ME, et al. Immune checkpoint inhibitor related myasthenia gravis: Single center experience and systematic review of the literature. J Immunother Cancer. 2019; 7: 319. 
31. Moreira A, Loquai C, Pföhler C, Kähler KC, Knauss S, Heppt MV, et al. Myositis and neuromuscular side-effects induced by immunecheckpoint inhibitors. Eur J Cancer. 2019; 106: 12-23.

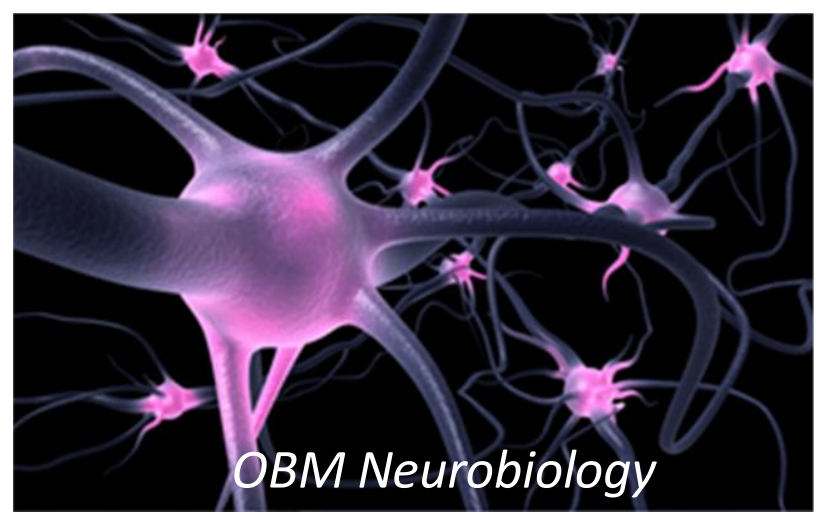

Enjoy OBM Neurobiology by:

1. Submitting a manuscript

2. Joining volunteer reviewer bank

3. Joining Editorial Board

4. Guest editing a special issue

For more details, please visit:

http://www.lidsen.com/journals/neurobiology 